\title{
Revista Ciencias de la Salud
}

\section{(2) MEDICINA}
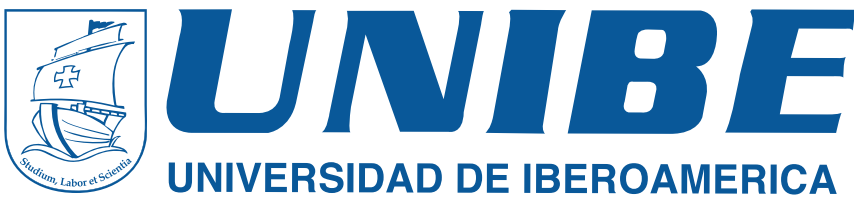

UNIVERSIDAD DE IBEROAMERICA

\section{Artículo}

\section{Uso de aspirina para prevención de preeclampsia}

\section{Use of aspirin for prevention of preeclampsia} Autores

Dra. Dayanna Villegas Chavarria

Médico General, egresada de la Universidad de Iberoamérica

Dra. Fabiola Cordero Espinoza

Médico General, egresada de la Universidad de Iberoamérica

Correspondencia: dayanavillegas15@hotmail.com

\section{Resumen}

La preeclampsia es una enfermedad multisistémica que puede traer repercusiones tanto en la madre como en feto, hasta el día de hoy el único tratamiento recomendado es la inducción del parto, es por esto que es importante buscar alternativas en cuanto al manejo, se ha observado un beneficio con el uso de aspirina en dosis bajas en mujeres de alto riesgo de desarrollar preeclampsia. La prevención de las secuelas asociadas, como el parto prematuro y el RCIU en el feto, o disfunción multiorgánica en la madre, se puede lograr al iniciar aspirina como profilaxis en $<16$ semanas de gestación.

\section{Palabras clave}

Aspirina, preeclampsia, prevención, embarazo.

\section{Abstract}

Preeclampsia is a multisystemic disease that can have repercussions in both the mother and the fetus. In the present, the only recommended treatment is induction of labor, which is why it is important to look for alternatives in terms of management. There is benefit with the use of low-dose aspirin in women at high risk of developing pre-eclampsia. Prevention of associated sequelae, such as preterm delivery and IUGR in the fetus, or multiple organ dysfunction in the mother, can be achieved by starting aspirin as prophylaxis at $<16$ weeks gestation.

\section{Keywords}

Aspirin, preeclampsia, prevention, pregnancy. Abreviaturas: RCIU (restricción del crecimiento intrauterino), DM (diabetes mellitus), American College of Obstetricians and Gynecologists (ACOG), United States Preventive Services Task Force (USPSTF), ciclooxigenasa (COX) 


\section{Justificación}

La preeclampsia es una de las complicaciones más comunes del embarzo con una prevalencia de $3-5 \%$. Este trastorno puede cursar con distintas complicaciones, ya sean maternas como insuficiencia hepática, insuficiencia renal, trastornos de la coagulación o complicaciones neurológicas, también se pueden presentar complicaciones fetales incluyendo restricción del crecimiento intrauterino, oligohidramnios, desprendimiento de placenta, ingreso a la unidad de cuidados intensivos neonatales, muerte fetal y muerte neonatal. El manejo de esta patología es inducción del parto, por lo tanto se puede incrementar la incidencia de parto prematuro y neonatos con bajo peso al nacer. $(1,2)$

Las investigaciones recientes han demostrado que utilizar aspirina en etapa temprana del embarazo, disminuye la incidencia de preeclampsia, por lo tanto disminuyen las repercusiones clínicas que pueden presentarse en la madre y el feto.

\section{Introducción}

La preeclampsia es un trastorno multisistémico, de etiología incierta, que se asocia con presión arterial elevada $>140 / 90 \mathrm{mmHg}$ asociado a proteinuria $>300 \mathrm{mg}$ en 24 horas. Ocurre posterior a las 20 semanas de embarazo. (1-4) Se define preeclampsia severa cuando cumple una o más de las siguientes características: hipertensión severa $(\geq 160 / \geq 110 \mathrm{~mm} \quad \mathrm{Hg})$, proteinuria severa ( $>5 \mathrm{~g} / 24$ horas), oliguria severa, trastornos cerebrales o visuales (dolor de cabeza, visión borrosa, escotomas), dolor en el cuadrante superior derecho, insuficiencia hepática (elevación de transaminasas) y trombocitopenia. (5)

Aunque se desconoce su etiología, esta enfermedad se caracteriza por la presencia de isquemia placentaria, activación del sistema inmune materno, aumento de la resistencia arterial, disminución en la producción de vasodilatadores y disfunción endotelial materna. (6)
En la actualidad, el único tratamiento es inducción del parto; sin embargo, esta práctica está relacionada con el parto prematuro y aumento del riesgo de morbilidad neonatal. Por lo tanto, se necesita reducir la incidencia de preeclampsia y de esta manera mejorar resultados maternos y neonatales. (2)

\section{Criterios de alto riesgo de preeclampsia} ACOG en una publicación reciente de Julio 2016, acepta la utilización de los factores de alto riesgo establecidos por el USPSTF: antecedentes de preeclampsia o hipertensión gestacional, hipertensión crónica, embarazos múltiples, enfermedad renal, diabetes tipo 1 o tipo 2 y enfermedades autoinmunes.

Criterios de riesgo moderado de preeclampsia Incluyen primer embarazo, edad materna $>35$ años, IMC $>30$, historia familiar de preeclampsia y características socioeconómicas. (7)

Resumen sobre recomendaciones para uso de baja dosis de aspirina

\begin{tabular}{|c|c|c|}
\hline Nivel de riesgo & $\begin{array}{l}\text { Factores de riesgo para } \\
\text { preeclampsia }\end{array}$ & Recomendación \\
\hline \multirow[t]{6}{*}{ Alto riesgo } & Historia de preeclampsia & \multirow{6}{*}{$\begin{array}{l}\text { Se recomienda } \\
\text { aspirina con solo } \\
1 \text { factor de alto } \\
\text { riesgo }\end{array}$} \\
\hline & Hipertensión crónica & \\
\hline & Antecedente DM1 o DM1 & \\
\hline & Enfermedad renal & \\
\hline & Gestación múltiple & \\
\hline & Enfermedad autoinmune & \\
\hline \multirow[t]{8}{*}{ Moderado riesgo } & Nuliparidad & \multirow{8}{*}{$\begin{array}{l}\text { Considerar } \\
\text { aspirina si } \\
\text { cumple algunos } \\
\text { criterios }\end{array}$} \\
\hline & $\mathrm{IMC}>30 \mathrm{~kg} / \mathrm{m}^{2}$ & \\
\hline & Edad $>35$ años & \\
\hline & $\begin{array}{l}\text { Historia familiar de } \\
\text { preeclampsia }\end{array}$ & \\
\hline & $\begin{array}{l}\text { Hijo previo con bajo peso } \\
\text { al nacer }\end{array}$ & \\
\hline & Raza negra & \\
\hline & $\begin{array}{ll}\text { Bajo } & \text { estatus } \\
\text { socioeconómico } & \end{array}$ & \\
\hline & $\begin{array}{l}10 \text { años desde el último } \\
\text { embarazo }\end{array}$ & \\
\hline Bajo riesgo & $\begin{array}{l}\text { No cumple ninguno de los } \\
\text { criterios anteriores }\end{array}$ & $\begin{array}{l}\text { No se recomien- } \\
\text { da aspirina }\end{array}$ \\
\hline
\end{tabular}

Fuente: Low-Dose Aspirin for the Prevention of Preeclampsia (14) 


\section{Mecanismo de acción de la aspirina en Preeclampsia}

La aspirina es un antiinflamatorio no esteroideo, que trabaja inhibiendo dos ciclooxigenasas (COX-1 y COX-2) que son necesarias para la síntesis de protaglandinas. La isoforma COX-1 está presente en el endotelio vascular y regula la producción de protaciclina y tromboxano A2, las mismas tienen efectos reguladores opuestos en la homeostasis vascular y función plaquetaria. La prostaciclina es un potente vasodilatador e inhibe la agregación plaquetaria, mientras que tromboxano A2 es un potente vasoconstrictor y promueve la agregación plaquetaria.

El efecto de la aspirina en la síntesis de prostaglandinas, es dependiente de la dosis. Una dosis baja (entre $60-150 \mathrm{mg} /$ día) acetila COX-1, resultando en la disminución en la producción de tromboxano A2, sin afectar la producción vascular de prostaciclina, restaurando así un equilibrio entre ambos. Asimismo, la aspirina parece mejorar los defectos del trofoblasto alterando la producción de citocinas específicas, disminuyendo la apoptosis y alteración de la agregación celular.

Además, en condiciones hipóxicas, la aspirina puede inhibir la expresión de tirosina quinasa 1 la cual muestra actividad pro-angiogénica.(3) Se sabe que la administración temprana de aspirina por su efecto anticoagulante y antiinflamatorio, inhibe la agregación plaquetaria y promueve la vasodilatación, por lo tanto mejora la formación y el desarrollo de la placenta, además aumenta el flujo sanguíneo a útero y placenta. $(4,8-10)$

\section{Efectos adversos de la aspirina}

Los efectos adversos más comunes documentados son úlcera gastrointestinal, sangrado, reacciones alérgicas, toxicidad hepática y renal; $\mathrm{y}$ neurotoxicidad. Se requiere monitoreo mientras se usa aspirina durante el embarazo. Es seguro utilizar una dosis de 60-150 $\mathrm{mg}$, el riesgo de sangrado aumenta significativamente cuando la dosis es superior a $150 \mathrm{mg}$.
No se ve aumentado el riesgo de desprendimiento de placenta ni hemorragia posparto.(11) Tampoco se asocia a anormalidades congénitas o el aumento de hemorragia intracraneal neonatal. $\mathrm{Y}$ al utilizarlo en el tercer trimestre no se observó evidencia de cierren en el ductus arterioso. $(3,12)$

Contraindicaciones para el uso de aspirina No se debe utilizar aspirina en pacientes con historia de alergia a la aspirina, hipersensibilidad a otros salicilatos o AINES. Se debe evitar bajas dosis de aspirina en pacientes con historia de pólipos nasales ya que puede causar broncoconstricción y poner en riesgo la vida, al igual que evitarse en pacientes con asma inducida por aspirina. Algunas contraindicaciones relativas incluyen historia de sangrado gastrointestinal, historia de sangrado genitourinario o disfunción hepática severa. (7)

\section{Métodos}

Para este artículo, se revisaron diferentes fuentes bibliográficas. Las principales fuentes de consulta fueron Pubmed, The New England Journal of Medicine y Elsevier. Los artículos elegidos fueron en su mayoría artículos de revisión y ensayos clínicos, con menos de 5 años de antigüedad. La búsqueda inicial arrojó $>100$ referencias bibliográficas; de las cuales mediante lectura del título y el abstract; se eligieron solamente 14 artículos.

\section{Discusión}

En una publicación del 2018, donde se incluyeron 1776 mujeres con embarazos únicos y de alto riesgo de preeclampsia, recibieron 150 mg de aspirina al día o placebo todas las noches desde la semana 11 a las 14 semanas hasta las 36 semanas de gestación. Se evidenció la reducción de preeclampsia en el grupo al que se le administró aspirina, en comparación con aquellas que recibieron placebo de 4.3 a $1.5 \%$. El máximo beneficio se obtuvo cuando se comienza la administración de aspirina antes de las 16 semanas de gestación. (5) 
Según Rolnik DL et al, en el ensayo doble ciego controlado con placebo, donde se abarcaron 13 maternidades de diferentes países, como España, Italia, Bélgica, Grecia e Israel. Se incluyeron 1591 pacientes con alto riesgo de desarrollar preeclampsia; de las cuales 806 recibieron placebo y 785 recibieron aspirina. Se administró $150 \mathrm{mg}$ diarios de aspirina de la semana 11-14 de gestación, hasta la semana 36 de gestación. Se mostró que en el grupo que recibió aspirina la incidencia de preeclampsia se disminuyó en un $62 \%$. Por lo tanto el ensayo evidenció un efecto beneficioso de la aspirina en la reducción de la tasa de preeclampsia. (10) \}

Del mismo modo, en la revista European Journal of Obstetrics Gynecology and Reproductive Biology, en un artículo publicado en el presente año, se realizó un estudio en Shangai, China; donde se incluyeron mujeres con alto riesgo y se observó que las dosis bajas de aspirina redujeron significativamente la incidencia de preeclampsia. Igualmente, se halló una disminución en la incidencia de RCIU, hemorragia postparto y parto prematuro. También, se comprobó mayor eficacia en aquellas pacientes con alta resistencia de la arteria uterina, por lo tanto, se recomienda realizar prueba Doppler, la cual es económica y accesible, como método de tamizaje, así de esta manera lograr determinar cuáles pacientes presentarían mayor beneficio al utilizar profilaxis con aspirina. (12)

\section{Conclusiones}

En conclusión, se demostró que la administración profiláctica de aspirina en el embarazo de mujeres de alto riesgo, puede reducir la incidencia de preeclampsia hasta es un $10 \%-50 \%$, al utilizar dosis de aspirina entre $60-150 \mathrm{mg}$ al día, a pesar de que aún no está claro la dosis exacta que se debe utilizar; el Colegio Americano de Obstetricia y Ginecología, recomienda el uso diario de $81 \mathrm{mg}$ de aspirina al día.
Se debe iniciar su uso entre la semana 12 y 28; preferiblemente antes de las 16 semanas de gestación, ya que la remodelación de las arterias espirales uterinas comienza a finales del primer trimestre y finaliza entre las 18 y 20 semanas de gestación, por lo tanto al concluir este periodo, la aspirina no tendría el mismo efecto. Asimismo, se aconseja recetar aspirina como profilaxis a mujeres con factores de alto riesgo de preeclampsia y una alta resistencia de la arteria uterina.

Finalmente, se evidenció que la aspirina reduce complicaciones mediadas por la placenta, como restricción del crecimiento fetal y parto prematuro. Por lo tanto, la aspirina es una alternativa en cuanto a la prevención de preeclampsia y sus complicaciones.

\section{Bibliografía}

1. Rahnemaei FA, Fashami MA, Abdi F, Abbasi M. Factors effective in the prevention of Preeclampsia:A systematic review. Taiwanase Journal of Obstetrics \& Gynecology. 2020;59(2):173-182.

2. Lin, L., Zhu, Y., Li, B. et al. Low-dose aspirin in the prevention of pre-eclampsia in China (APPEC study): protocol for a multicentre randomized controlled trial. Trials 19, 608 (2018).

https://doi.org/10.1186/s13063-018-2970-3

3. Henderson JT, Whitlock EP, O'Connor E, Senger CA, Thompson JH, Rowland MG. Low-dose aspirin for prevention of morbidity and mortality from preeclampsia: a systematic evidence review for the U.S. Preventive Services Task Force. Ann Intern Med. 2014;160;695-703.

4. Leavitt K, Običan S, Yankowitz J. Treatment and Prevention of Hypertensive Disorders During Pregnancy. Clinics in Perinatology. 2019;46(2):173-185. 
5. Lisa Story, Catherine Nelson-Piercy. Aspirin versus placebo in pregnancies at high risk for preterm pre-eclampsia. Obstetric Medicine. 2018;11(2):90-99.

6. Montfort P, Scheepers HCJ, Dooren IMA, Meertens LJE, Zelis M, Zwaan IM, et al. Low-dose aspirin usage among women with an increased preeclampsia risk: A prospective cohort study. Acta Obstetricia et Gynecologica Scandinavica. 2020; 00:1-9.

7. Roberge S, Nicolaides K, Demers S, Hyett J, Chaillet N, Bujold E. The role of aspirin dose on the prevention of preeclampsia and fetal growth restriction: systematic review and metaanalysis. American Journal of Obstetrics \& Gynecology. 2017;216(2):110-120.

8. American College of Obstetricians and Gynecologists. Low-Dose Aspirin Use During Pregnancy. Committee Opinion. 2018; 132(1):44-52.

9. Cui Y, Zhu B, Zheng F. Low-dose aspirin at $\leq$ 16 weeks of gestation for preventing preeclampsia and its maternal and neonatal adverse outcomes: A systematic review and meta-analysis. Experimental and Therapeutic Medicine. 2018;15(5):4361-4369.

10. Rolnik DL, Wright D, Poon LC, O'Gorman N, Syngelaki A, de Paco Matallana C, et al. Aspirin versus Placebo in Pregnancies at High Risk for Preterm Preeclampsia. New England Journal of Medicine. 2017;377(7):613-622.

11. Alcalá Lorente M, Navarro Rubio G. Prevención de la preeclampsia con aspirina. Progresos de Obstetricia y Ginecología. 2015:1-5.

12. Gu W, Lin J, Hou Y-Y, Lin N, Song M-F, Zeng W-J, et al. Effects of low-dose aspirin on the prevention of preeclampsia and pregnancy outcomes: A randomized controlled trial from Shanghai, China.

European Journal of Obstetrics Gynecology and Reproductive Biology. 2020;248:156-163.
13. Roberge S, Bujold E, Nicolaides KH. Aspirin for the prevention of preterm and term preeclampsia: systematic review and metaanalysis. American Journal of Obstetrics \& Gynecology. 2018;218(3):287-293.

14. Fantasia HC. Low-Dose Aspirin for the Prevention of Preeclampsia. Nursing for Women's Health. 2018;22(1):87-92. 